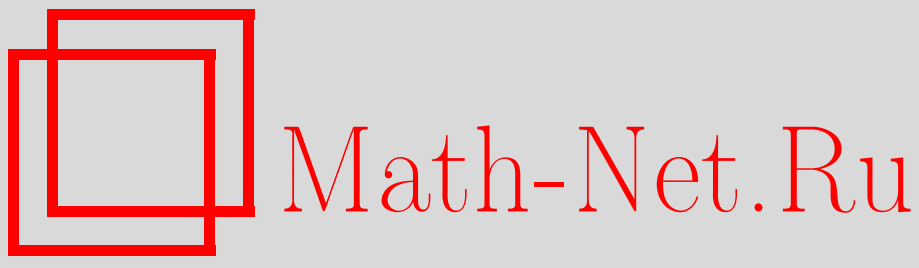

А. В. Матвеев, О связи неприводимых представлений алгебр Ли с неприводимыми представлениями $p$-групп, Матем. сб., 1996, том 187, номер 7, 93-96

DOI: https://doi.org/10.4213/sm146

Использование Общероссийского математического портала Math-Net.Ru подразумевает, что вы прочитали и согласны с пользовательским соглашением http://www.mathnet.ru/rus/agreement

Параметры загрузки:

IP : 52.205 .19 .152

26 апреля 2023 г., $12: 56: 10$ 
УДК 512

\author{
А.В. Матвеев
}

\title{
О связи неприводимых представлений алгебр Ли с неприводимыми представлениями $p$-групп
}

\footnotetext{
В работе доказьвается теорема о соответствии между неприводимьми комплексными представлениями конечной $p$-группы и неприводимьми представлениями ассоциированной с ней нильпотентной алгебры Ли над полем характеристики $p$. В качестве следствия получается совпадение множеств степеней неприводимых представлений.
}

Библиография: 4 названия.

В этой заметке изучается вопрос о связи между неприводимьми представлениями $p$-группы и ассоциированной с ней алгебры Ли над полем $\mathbb{Z}_{p}$. Постановка такой задачи принадлежит А.И. Кострикину. Существует предположение, что наборы размерностей неприводимых представлений $p$-группы и ассоциированной с ней алгебры Ли совпадают. Некоторые результаты по этому поводу были получены в [3]. Здесь эти результаты обобщаются.

Пусть $L$ - конечномерная алгебра Ли над $\mathbb{Z}_{p}$, класса нильпотентности $c<p$. Определим на $L$ с помощью формулы Кэмпбелла-Хаусдорфа групповую операцию и обозначим полученную группу $L^{\circ}$. Очевидно, что $L^{\circ}-p$-группа (конечная) экспоненты $p$ и класса нильпотентности $c$, если ступень нильпотентности $L$ есть $c-1$. Очевидно также, что если $P$ - подалгебра в $L$, то $P^{\circ}-$ подгруппа в $L^{\circ}$, причем, если $P$ - идеал, то $P^{\circ}$ - нормальная подгруппа в $L^{\circ}$. Верно также и обратное утверждение. Кроме того, для членов нижнего центрального ряда алгебры Ли $L$ и групшы $L^{\circ}$ имеем $\left(L^{k}\right)^{\circ}=\gamma_{k}\left(L^{\circ}\right)$ (см. [2]).

Нам потребуются некоторые понятия и утверждения из [1]. Пусть $L-$ конечномерная разрешимая $p$-алгебра Ли над алгебраически замкнутым полем характеристики, большей двух, $L^{*}-$ пространство линейных функций на $L$.

ОПРЕДЕЛЕНИЕ 1 . Пусть $l \in L^{*}$, тогда $p$-подалгебра Ли $P$ называется $n о л я p u-$ защией для $l$, если:

(a) $l\left(P^{2}\right)=\{0\}$,

(b) $P$ имеет наибольшую размерность среди всех подалгебр, удовлетворяющих пункту (a).

ОПРЕДЕЛЕНИЕ 2. Если $l \in L^{*}$, то $p$-подалгебра Ли $P$ в $L$ назьвается $V$-noляризащией для $l$, когда существует цепь подалгебр

$$
\{0\} \subset K_{0} \subset \cdots \subset K_{n}=P=G_{n} \subset \cdots \subset G_{0}=L
$$

таких, что

(a) $K_{i} \triangleleft G_{i}$ и $l\left(K^{2}\right)=\{0\}$,

(b) $G_{i+1}=\left\{g \in G_{i} \mid l([f, g])=0 \forall f \in K_{i}\right\}$. 
Пусть $P(l)$ - множество поляризаций для $l, P_{V}(l)$ - множество $V$-поляризаций для $l$. Пусть $s \in L^{*}$, тогда через $P(l, s)$ обозначим множество $\{P \in P(l) \mid$ $\left.l^{p}(f)-l\left(f^{[p]}\right)=s^{p}(f) \forall f \in P\right\} ; P_{V}(l, s) \stackrel{\text { def }}{=} P(l, s) \cap P_{V}(l)$. Верно, что для любой $P \in P_{V}(l), P(l)$ существует $s \in L^{*}$ такая, что $P \in P_{V}(l, s), P(l, s)$, соответственно. В [1] доказаны следующие утверждения.

УТВЕРЖДЕНИЕ 1. $P_{V}(l) \neq \varnothing u P_{V}(l) \subset P(l)$ для любой формы $l \in L^{*}$. Пусть $\widetilde{M}(l, s, P) \stackrel{\text { def }}{=} U(L, s) \otimes_{U\left(P,\left.s\right|_{P}\right)}(K u)^{\lambda}$ - подкрученный индуцированный $L$-модуль, где $\lambda=\frac{1}{2} \operatorname{tr}(\operatorname{ad} L / P), a(K u)^{\lambda}-$ одномерный $P$-модуль, определяемый следующим образом:

$$
f \circ u \stackrel{\text { def }}{=}(l(f)+\lambda(f)) u \text { для любого элемента } f \in P .
$$

\section{УТВЕРЖ ДЕНИЕ 2.}

(а) Eсли $P \in P_{V}(l, s)$, то $\widetilde{M}(l, s, P)$ - неприводимый $L$-модуль такой, что $\operatorname{dim} \widetilde{M}(l, s, P)=p^{\operatorname{dim}(L / P)}$.

(b) Eсли $M$ - неприводимый $L$-модуль, то существуют $l, s \in L^{*}$ u $P \in$ $P_{V}(l, s)$ maкие, что $M \cong \widetilde{M}(l, s, P)$.

УТВЕРЖДЕНИЕ 3. Если $P \in P(l, s) u Q \in P_{V}(l, s), m o \widetilde{M}(l, s, P) \cong \widetilde{M}(l, s, Q)$.

Обозначим групповой коммутатор $(\cdot, \cdot)$, лиевский $-[\cdot, \cdot]$. Пусть $\mu \in L^{*}$; определим $\widetilde{\mu}: P^{\circ} \rightarrow \mathbb{C}^{*}, \widetilde{\mu}(x)=\varepsilon^{\mu(x)}$ для любого $x \in L^{\circ}$, где $\varepsilon-$ первообразный корень $p$-й степени из единицы.

Teорема. 1. Eсли $P \in P_{V}(\mu)$, mо $P^{\circ}$ - nодгрупnа в $L^{\circ}$ такая, что представление $L^{\circ}$, индуцированное $с \widetilde{\mu}: P^{\circ} \rightarrow \mathbb{C}^{*}$, неприводимо.

2. Если $M$ - неприводимыи $L^{\circ}$-модуль, соответствующий некоторому ненулевому представлению, то существует подалгебра $P \subset L$ такая, что

(а) $M$ изоморфен модулю, индуцированному с некоторого представления $l: P^{\circ} \rightarrow \mathbb{C}^{*}$

(b) существует форма $\mu \in P^{*}$ такая, что $l=\widetilde{\mu}, u$ для любой $\mu^{\prime} \in L^{*}$, являющейся линейным продоләсением $\mu, P \in P\left(\mu^{\prime}\right)$.

ДокАЗАТЕЛьство. 1. По критерию Макки представление, индуцированное с $\tilde{\mu}$, неприводимо тогда и только тогда, когда для каждого $x \notin P^{\circ}$ существует элемент $y \in x H x^{-1} \cap H$ такой, что $\widetilde{\mu}(y) \neq \widetilde{\mu}\left(x^{-1} y x\right)$. Предположим, что условия критерия Макки не выполнены, а именно, сушествует $x \in L \backslash P$ такой, что $\widetilde{\mu}((x, p))=1$ или, что то же самое, $\mu((x, p))=0$ для любых $p \in P$ таких, что $(x, p) \in P^{\circ}$. Тогда существует $i$ такое, что $x \in G_{i}, x \neq G_{i+1}$, и из определения $V$-поляризации следует, что сушествует $y \in K_{i}$ такой, что $\mu([x, y]) \neq 0$.

Поскольку для любых $a_{1}, a_{2}, \ldots, a_{n}$ справедливо

$$
\left(a_{1}, a_{2}, \ldots, a_{n}\right)=\left[a_{1}, a_{2}, \ldots, a_{n}\right]+b, \quad b \in L^{n+1}, \quad n=2,3, \ldots,
$$

при одной и той же расстановке скобок, то верно также, что $[x, y]=(x, y)+\sum k_{j} z_{j}$, где $k_{j} \in \mathbb{Z}_{p}, z_{j}$ - групповые коммутаторы от $x, y$ веса больше 2 . 
Любой групповой коммутатор от $x, y$ принадлежит $K_{i}$, так как $K_{i} \triangleleft G_{i}$. Тогда $\mu\left(z_{j}\right)=0$ для любого коммутатора $z_{j}$, у которого ни один из последних сомножителей не есть $x$, поскольку $\mu\left(K_{i}^{2}\right)=\{0\}$. В силу предположения $\mu\left(z_{j}\right)=0$ для остальных коммутаторов $z_{i}$ и $\mu((x, y))=0$.

Так как $\mu((x, y))=\mu\left(z_{j}\right)=0, \mu([x, y]) \neq 0$, получаем противоречие с полученным выше равенством.

2. Приступим к доказательству части 2 теоремы.

УТВЕРЖДЕНИЕ. В условиях части 2 теоремь существуют подгруппы $G_{0}, \ldots, G_{n} ; K_{0}, \ldots, K_{n} ; T$ группь $L^{\circ}$ и представление $l: T \rightarrow \mathbb{C}^{*}$ такие, что

(a) $K_{n}=T=G_{n} \subset \cdots \subset G_{0}=L^{\circ}$,

(b) $K_{i} \triangleleft G_{i}, K_{i} \subseteq T, i=0, \ldots, n$,

(c) $l\left(\gamma_{2}\left(K_{i}\right)\right)=\{1\}$,

(d) $G_{i+1}=\left\{g \in G_{i} \mid l((f, g))=1 \forall f \in K_{i}\right\}$,

(е) $M$ изоморфен модулю, индуцированному с $l$.

ДокАЗАТЕЛЬСТво. Будем использовать следующий результат.

ПРЕДЛОЖЕНИЕ [4, с. 530]. Пусть $G$ - неабелева сверхразрешимая группа, тогда существует нормальная абелева подгруппа, которая собственным образом содержит центр.

Построим подг руппы $G_{1}$ и $K_{0}$.

Предположим вначале, что представление, соответствуюшее модулю $M,-$ точное. Пусть $K_{0}$ - нормальная абелева подгруппа, собственньм образом содержашая центр группы $L^{\circ}$. Рассмотрим $M$ как $K_{0}$-модуль. Модуль $M$ является прямой сум-

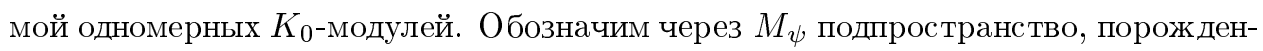
ное всеми одномерньми $K_{0}$-подмодулями, имеющими характер $\psi$. Тогда получаем разложение в прямую сумму подпространств $M=\sum M_{\psi}$.

Пусть $v \in M_{\psi}$, тогда для любых $f \in K_{0}, g \in L^{\circ}$ имеем:

$$
f(g v)=g\left(g^{-1} f g\right) v=g \psi\left(g^{-1} f g\right) v=\psi_{g}(f) g v,
$$

где $\psi_{g}$ - функция на $K_{0}$, определяемая равенством $\psi_{g}(f) \stackrel{\text { def }}{=} \psi\left(g^{-1} f g\right)$.

Пользуясь тем, что представление точное, можно показать, как в [4, с. 532], что $M \neq M_{\psi}$. Несложно показать также, что элементы $L^{\circ}$ транзитивно переставляют подпространства $M_{\psi}$.

Пусть $M_{1}=M_{\psi_{1}}$, где $\psi_{1}$ - некоторый фиксированный характер $K_{0}$-модуля $M$. Определим подгруппу

$$
G_{1} \stackrel{\text { def }}{=}\left\{g \in L^{\circ} \mid g M_{1}=M_{1}\right\}=\left\{g \in L^{\circ} \mid \psi_{1}\left(g^{-1} f g\right)=\psi_{1}(f) \forall f \in K_{0}\right\} .
$$

Предположим теперь, что представление, соответствуюшее модулю $M$, не является точным, и $N \triangleleft L^{\circ}$ - ядро этого представления. Соответствующее представление групшы $G / N$ будет точньм. Определим для него подгруппы $\widetilde{G}_{1}, \widetilde{K}_{0}$, функцию $\widetilde{\psi}_{1}$ и подпространство $M_{1}$, как мы это сделали в случае точного представления. Возьмем в качестве $G_{1}, K_{0}, \psi_{1}$ прообразы для $\widetilde{G}_{1}, \widetilde{K}_{0}, \widetilde{\psi}_{1}$ при каноническом гомоморфизме $\varphi: G \rightarrow G / N$. 
В любом случае верно следующее: $K_{0} \triangleleft L^{\circ}, G_{1}=\left\{g \in L^{\circ} \mid \psi_{1}\left(g^{-1} f g\right)=\right.$ $\left.\psi_{1}(f) \forall f \in K_{0}\right\}, G_{1} \neq L^{\circ}, M_{1}$ - неприводимый $G_{1}$-модуль, $L^{\circ}$-модуль $M$ индуцирован с $M_{1}$ (см. [4, с. 532]).

Если $\operatorname{dim}\left(M_{1}\right) \neq 1$, то к $G_{1}, M_{1}$ можно применить те же рассуждения, построив таким образом $K_{1}, G_{2}, M_{2}$, и т. д. В итоге получим: $T \stackrel{\text { def }}{=} G_{n} \subset G_{n-1} \subset \cdots \subset$ $G_{1} \subset G_{0} \stackrel{\text { def }}{=} L^{\circ}, K_{i} \triangleleft G_{i}, M_{n} \subset M_{n-1} \subset \cdots \subset M_{1} \subset M_{0} \stackrel{\text { def }}{=} M$, где $\operatorname{dim}\left(M_{n}\right)=1$ и $L^{\circ}$-модуль $M$ индуцирован с $T$-модуля $M_{n}$.

Для любых $i, j=0, \ldots, n$ имеем $K_{i} \subseteq G_{j}$ (для $i \geqslant j$ это очевидно, для $i<j$ это доказывается индукцией), в частности, $K_{i} \subseteq T$ для любого $i$. Пусть $l \stackrel{\text { def }}{=} \psi_{n}$. Очевидно, что $\psi_{j}=\left.l\right|_{K_{j-1}}$, где $j=1, \ldots, n$, поэтому:

$$
\begin{aligned}
G_{i+1} & \stackrel{\text { def }}{=}\left\{g \in G_{i} \mid \psi_{i+1}\left(g^{-1} f g\right)=\psi_{i+1}(f) \forall f \in K_{i}\right\} \\
& =\left\{g \in G_{i} \mid l((f, g))=1 \forall f \in K_{i}\right\} .
\end{aligned}
$$

Таким образом, утверждение доказано.

Рассмотрим теперь подалгебру Ли $P$ в $L$ такую, что $T=P^{\circ}$. Пусть $l: P^{\circ} \rightarrow \mathbb{C}^{*}$ - одномерное представление, определим функцию $\mu: P \rightarrow \mathbb{Z}_{p}$ так, что $l(f)=\varepsilon^{\mu(f)}$ для $p \in P$. Легко показать, что функция $\mu$ линейна.

Рассмотрим функцию $l$, построенную в утверждении. Так как $l((f, g))=1$ равносильно тому, что $\mu((f, g))=0$, то, аналогично доказательству части 1 теоремы, можно показать (поменяв местами групповой и лиевский коммутаторы), что $P$ максимальное вполне изотропное подпространство относительно билинейной формы $B(x, y) \stackrel{\text { def }}{=} \mu^{\prime}([x, y])$. Показьвается, что для любого $x \in L \backslash P$ сушествует элемент $p \in P$ такой, что $[x, y] \in P$ и $\mu([x, y]) \neq 0$.

Таким образом, $P$ - поляризация и теорема доказана.

Используя приведенные выше утверждения из [1] и нашу теорему, получаем

СлЕДСТвИЕ. Множество степеней неприводимъх представлений над $\mathbb{C}$ группь $L^{\circ}$ совпадает с множеством степеней абсолютно неприводимых представлений над $\mathbb{Z}_{p}$ алгебры Ли $L$.

Пользуясь случаем, я хотел бы выразить благодарность Ю.А. Бахтурину за помощь в работе над этой заметкой.

\section{Список литературы}

1. Strade H. Darstellungen auflösbarer Lie-p-Algebren // Math. Ann. 1978. V. 232. №1. P. $15-32$.

2. Андреев K. K. Некоторые достаточные условия локальной нильпотентности в группах // Труды Моск. ин-та электр. машиностр. 1974. №30. С. 19-31.

3. Басманова С. А. О связи между неприводимьми представлениями конечной $p$-группы и ассоциированной с ней алгеброй Ли // Вестн. МГУ. Сер. 1. Матем., мех. 1986. № 5. C. $58-60$.

4. Ленг С. Алгебра. М.: Мир, 1968. 